\title{
Presentation and Management of an Interprosthetic Femo- ral Fracture between a Total Hip Arthroplasty and Revised Total Knee Arthroplasty with Distal Femoral Replacement
}

\author{
Mohamed Shaalan, MD, MRCS* (D), Ashraf Dawood, MD, FRCS and William Gaine, MD, FRCS \\ Orthopaedic Department, Sligo University Hospital, Ireland

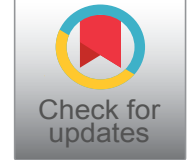

*Corresponding author: Mohamed Shaalan, MD, MRCS, Orthopaedic Department, Sligo University Hospital, Ireland

\begin{abstract}
An 83-year-old female sustained a right femoral interprosthetic fracture following a fall, She had a high BMI with a background history of ischaemic heart disease and type II diabetes. She was placed in a Thomas splint and underwent surgical fixation with a plate on the following trauma list.

She was non-weight bearing for six weeks before partial weight bearing was commenced when callus appeared on $X$-ray. At six months good callus was evidenced and she was fully weight bearing.
\end{abstract}

\section{Background}

Interprosthetic (IP) femur fractures occur between a total hip arthroplasty (THA) and a total knee arthroplasty (TKA) and complicate approximately $1.25 \%$ to $8.8 \%$ of ipsilateral hip and knee arthroplasties [1].

More recent reports estimate the risk to be about $5-7 \%$ of all periprosthetic fractures [2].

Regarding interprosthetic distance, there is no clear consensus on how far apart hip and knee stems should be to mitigate fracture risk. A reduced distance may lead to higher stress concentration with the increased risk for fracture at this location [3].

Classification of interprosthetic fractures has been described as follows:

Type I: Fracture around a femoral prosthesis,

Type II: Fracture around a knee prosthesis without a stem,

Type III: Fracture around a knee prosthesis that con- tain a stem extension.

Type I and II fractures are further subdivided into groups A (stable femoral and knee prosthesis), B (unstable femoral but stable knee prosthesis), C (stable femoral but unstable knee prosthesis), and D (unstable femoral and knee prostheses).

Type IIIA represents stable prostheses with viable bone between the prostheses. Type IIIB describes stable femoral and knee prostheses with a nonviable fragment or lack of bone between prostheses ends. Type IIIC describes unstable prostheses (hip, knee, or both) with viable bone between the prostheses, and Type IIID represents unstable prostheses (hip, knee, or both) with a nonviable interval fragment due to lack of viable bone between prostheses' ends $[3,4]$.

\section{Case Presentation}

An 83-year-old female presented with an interprosthetic fracture right femur after a fall.

On clinical examination, there is deformity of the right thigh and the leg was held in external rotation. She had good distal pulses and no neurological deficits. A Thomas splint was applied after a tensor fascia lata block. Her medical conditions included a high BMI, ischaemic heart disease and type II diabetes.

The following day she underwent open reduction and internal fixation with a Kinamed Plate and supracables through a subvastus approach. The fracture was determined to be a Type IIIC according the Pires classification occurring at the tip of the distal prosthetic stem 

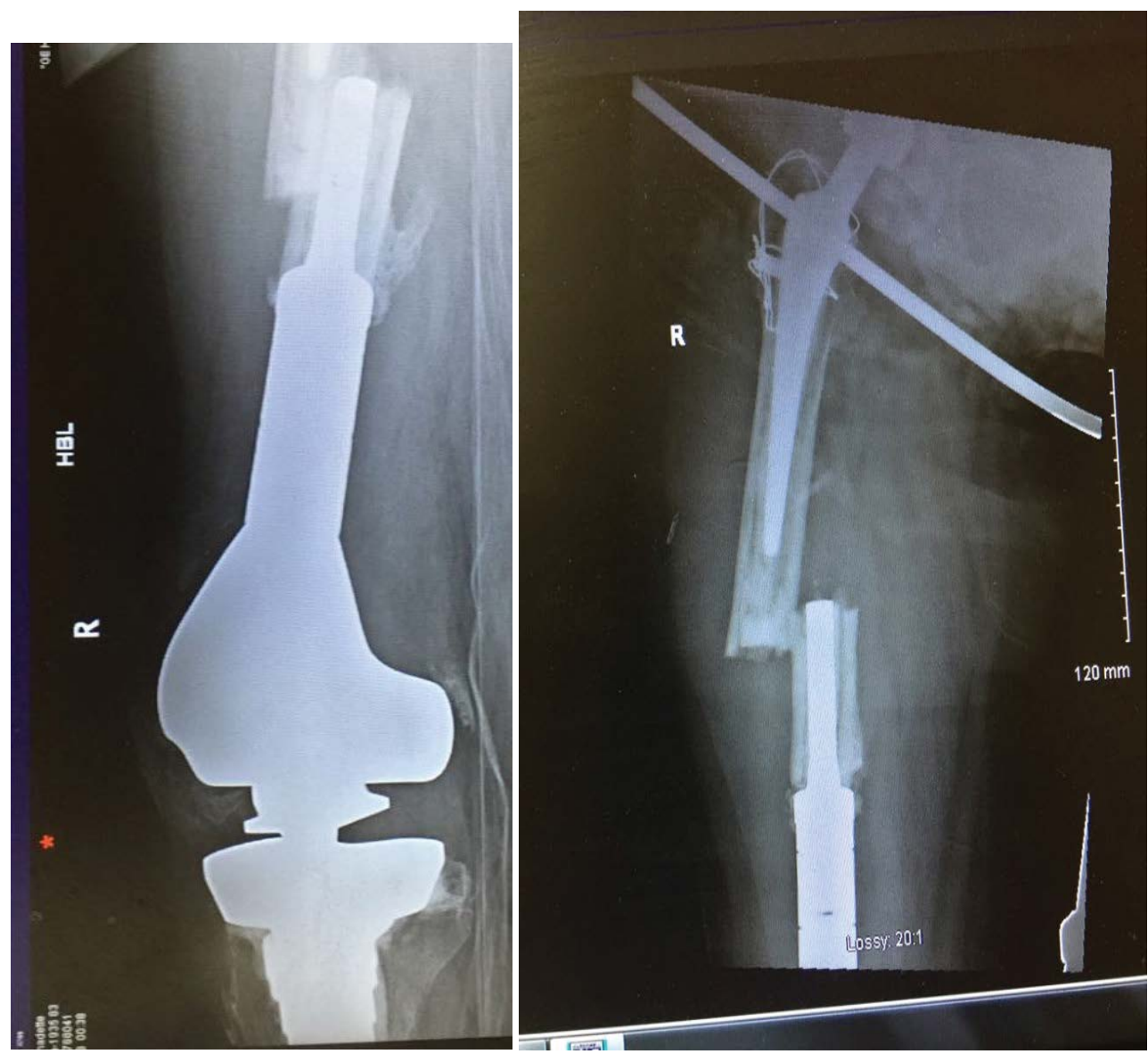

Figure 1: Type IIIC fracture according Pires classification occurring at the tip of the distal prosthetic stem.

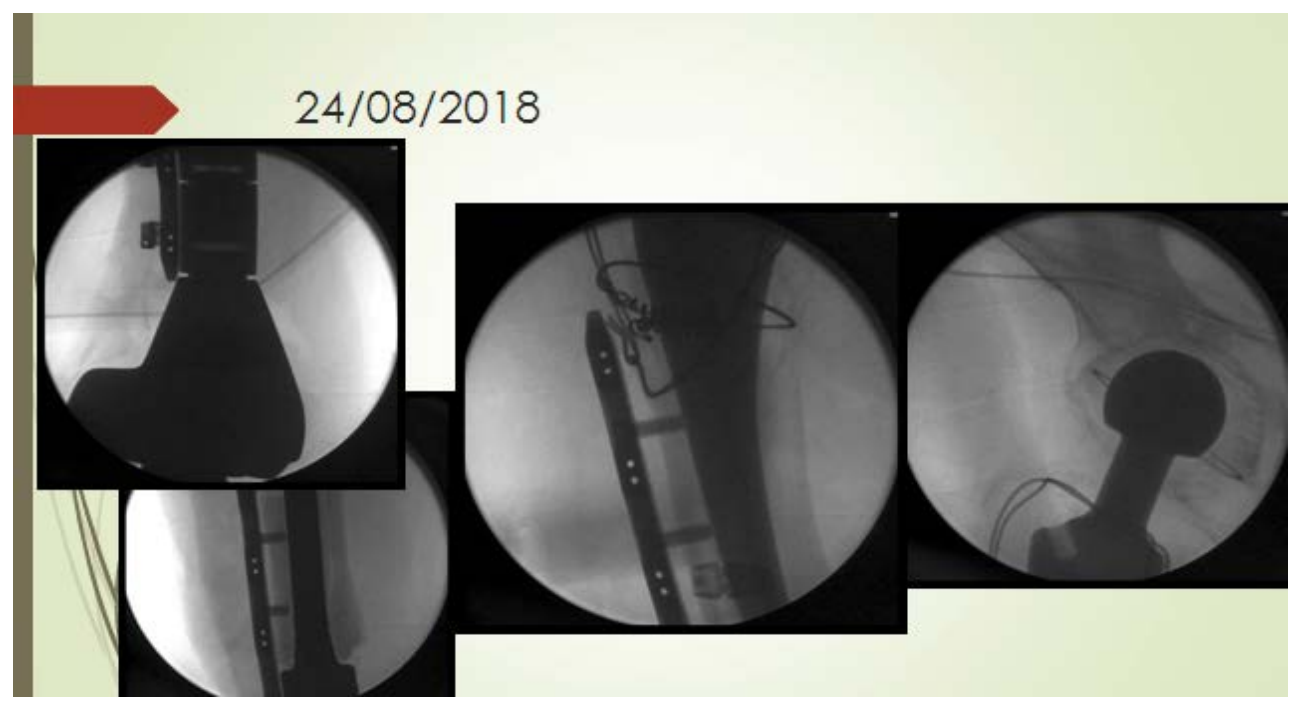

Figure 2: Intraoperative imaged.

(Figure 1). The fixation construct was stable (Figure 2). The fixation was supplement with 15 grams hydroxyapatite bone graft granules around the fracture site. The patient was mobilized non weight bearing for six weeks. Partial weight bearing was commenced at six weeks and full weight bearing at 10 weeks (Figure 3 and Figure 4).

The patient was able to dispense with walking aids at six months. She regained a good range of motion of the hip and knee and achieved a good functioning pain-free result.

\section{Discussion}

Interprosthetic femur fractures represent a difficult clinical problem with a growing incidence in the face of more patients living with ipsilateral total hip and knee arthroplasties.

On a review of the literature, Soenen, et al. in a multicenter retrospective series with 14 interprosthetic femoral fracture, none of the six fractures proximal to a constrained TKR with long stem achieved union by 


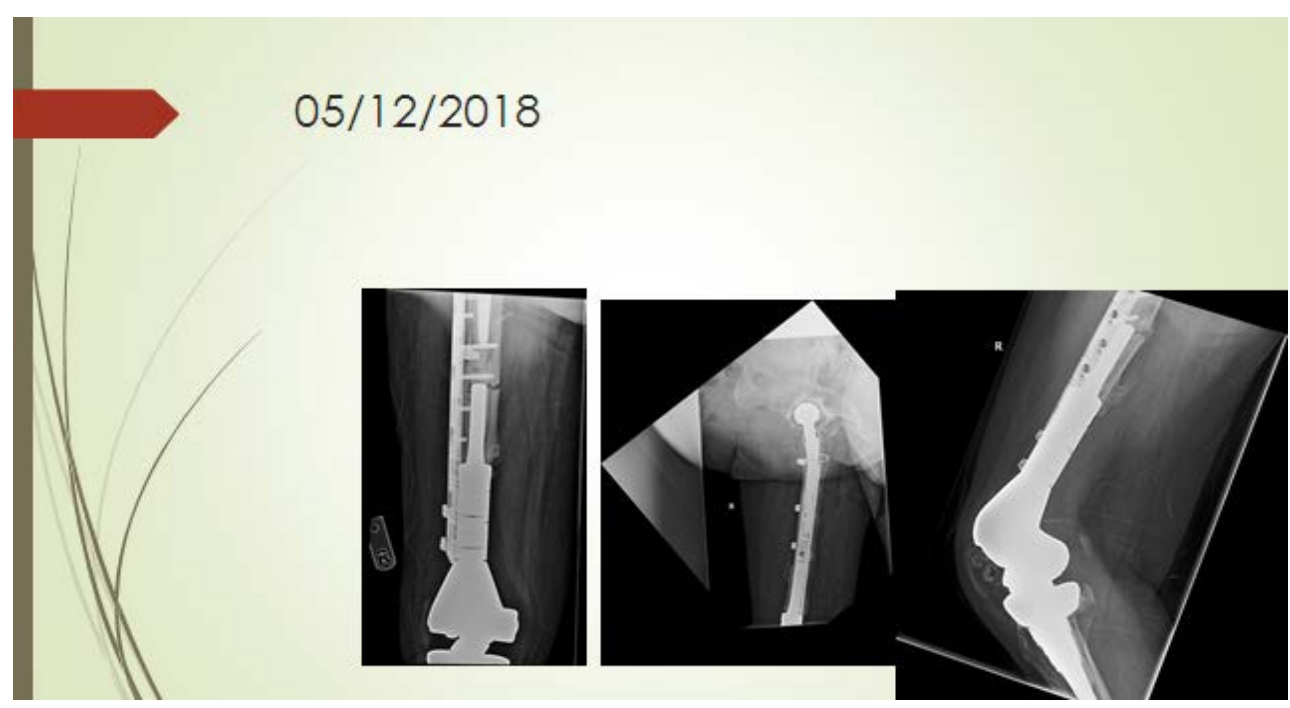

Figure 3: F/u X-ray.

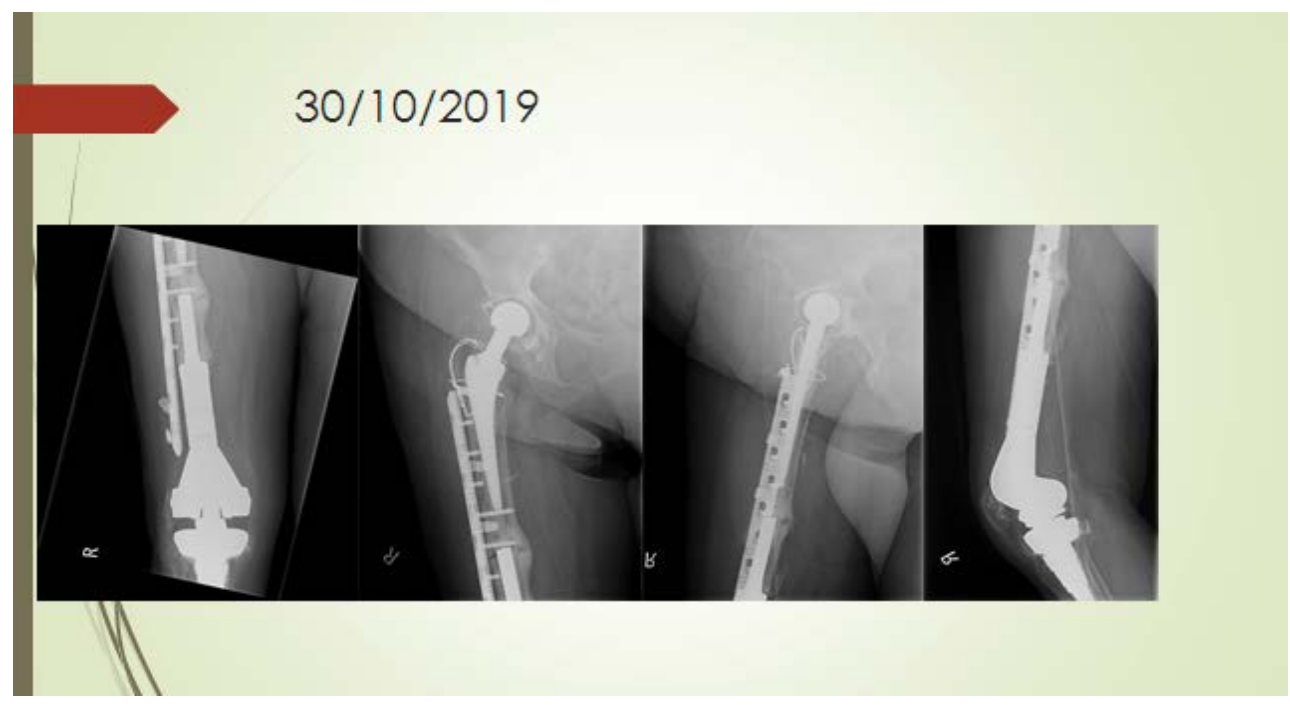

Figure 4: F/U X-ray.

primary intention, whereas seven of the eight type-C fractures did so. Finally, 12 cases showed favorable evolution, with three secondary total femur replacements (TFR) and one death at 6 months without bony union or revision and one patient waiting for TFR [5].

\section{Conclusion}

Interprosthetic fracture is challenging presentation. The use of a locking Kinamed plate supplemented by cerclage cables has been a successful method of fixation in the case described.

\section{References}

1. Valle Cruz JA, Urda AL, Serrano L, Rodriguez-Gonzalez FA, Otero J, et al. (2016) Incidence of and risk factors for femoral fractures in the gap between hip and knee implants. Int Orthop 40: 1697-1702.

2. Bonnevialle $P$, Marcheix PS, Nicolau $X$, Arboucalot $M$, Lebaron $M$, et al. (2019) Interprosthetic femoral fractures: Morbidity and mortality in a retrospective, multicenter study. Orthop Traumatol Surg Res 105: 579-585.
3. Pires RES, De Toledo Lourenço PRB, Labronici PJ, Rosa da Rocha L, Balbachevsky D, et al. (2014) Interprosthetic femoral fractures: Proposed new classification system and treatment algorithm. Injury 45: S2-S6.

4. Pires RES, Silveira MPS, Da Silva Resende AR, Santana Junior EO, Oliveira Campos TV, et al. (2017) Validation of a new classification system for interprosthetic femoral fractures. Injury 48: 1388-1392.

5. Soenen M, Mihaud H, Bonnomet F, Girard J, Mathevon H, et al. (2011) Interprosthetic femoral fracture: Analysis of 14 cases. Proposal for an additional grade in the Vancouver and SoFCOT classifications. Orthop Traumatol Surg Res 97: 693-698. 